\title{
Effect of combined endurance-resistance training and soy extract supplementation on expression of eNOS gene in ovariectomized rats
}

\author{
Morteza Jourkesh ${ }^{1}$, Siroos Choobineh², Rahman Soori², Ali Asghar Ravasi ${ }^{2}$
}

\begin{abstract}
${ }^{1}$ Department of Exercise Physiology, Faculty of Physical Education and Sport Sciences, University of Tehran, Aras International Campus, Tehran, Iran

2Department of Exercise Physiology, Faculty of Physical Education and Sport Sciences, University of Tehran, Tehran, Iran
\end{abstract}

Submitted: 27 July 2017

Accepted: 10 September 2017

Arch Med Sci Atheroscler Dis 2017; 2: e76-e81

DOI: https://doi.org/10.5114/amsad.2017.70714

Copyright $\odot 2017$ Termedia \& Banach

\section{Abstract}

Introduction: Menopause is an independent risk factor for cardiovascular disease (CVD). Physical exercise and soybean diets have been suggested to reduce the risk of CVD in postmenopausal women. The purpose of this study was to investigate the effects of combined resistance and endurance (RE) training and soy extract (SOY) supplementation, both known to improve endothelial function, on expression of the eNOS gene in the heart of ovariectomized $(\mathrm{OVX})$ rats.

Material and methods: Fifty female Wistar rats were divided into five groups: 1) sham (SHAM); 2) ovariectomy (OVX); 3) ovariectomy with soy extract supplementation (OVX + SOY); 4) OVX with RE training (OVX + RE); 5) and ovariectomy plus RE training with soy extract supplementation (OVX $+\mathrm{RE}+\mathrm{SOY}$ ). RE training and soy extract supplementation were administered alone or in combination for 6 weeks. The effects of these treatments on cardiac eNOS expression were measured using real-time PCR.

Results: Ovariectomy down-regulated cardiac eNOS gene expression; however, 6 weeks of SOY treatment or RE training reversed this effect $(p \leq 0.05)$. The combination of SOY plus RE was greater than RE or SOY alone in reversing estrogen-deficiency-caused eNOS down-regulation $(p \leq 0.05)$.

Conclusions: Our data suggest that the combinatory regimen of soy extract supplementation and regular RE training may be more beneficial to cardiovascular disease risk in a menopause rat model than either exercise or soy supplementation alone.

Key words: exercise training, ovariectomy, phytoestrogen, eNOS, soy extract.

\section{Introduction}

Endothelial-derived nitric oxide (NO), synthesized by endothelial NO synthase (eNOS) from amino acid L-arginine and molecular oxygen, plays a pivotal role in maintaining vascular homeostasis [1]. A decline of eNOS activity and/or expression is associated with cardiovascular diseases such as hypertension [2, 3], atherosclerosis [4], and stroke [5]. Cardiovascular disease (CVD) is more prevalent in women after menopause [6], but develops in women on average 10 years later in life compared with men. This lag has been attributed to the protective effects of female

\author{
Corresponding author: \\ Assoc. Prof. Siroos Choobineh \\ $\mathrm{PhD}$ \\ Assoc. Prof. Rahman Soori \\ $\mathrm{PhD}$ \\ Department of \\ Exercise Physiology \\ Faculty of Sport \\ and Exercise Sciences \\ Tehran University \\ 14398-13117 \\ Tehran, Iran \\ E-mail: \\ Choobineh@ut.ac.ir, \\ Soori@ut.ac.ir \\ mjourkesh@ut.ac.ir
}


sex hormones, particularly estrogens [7], which dip in the years after menopause. Accordingly, vascular protective effects of estrogen by various mechanisms have also been reported [8]. Observations of hormone replacement therapy (HRT) in relation to incidence of CVD originally suggested a reduced incidence of CVD [9], but the use of HRT as secondary prevention for CVD is now not recommended, because more recent studies have shown that HRT could have significant side effects in women [10]. Nevertheless, more experimental work on the relationship between estrogen and markers of CVD is warranted.

The biological activities [11-16] and beneficial effects of isoflavones in preventing various chronic diseases including CVD have been documented $[17,18]$. Soybeans are a common dietary source of isoflavones, a class of phytoestrogens [19], and the predominant soy isoflavones are genistein, daidzein, and, in lower concentration, glycitein [20]. Soy isoflavones have favorable effects on postmenopausal women with type 2 diabetes [21], and in non-ovariectomized rodents, a genistein-containing diet for 1 month $(600 \mathrm{mg}$ genistein/kg diet) induces beneficial effects on cardiovascular risk factors and aortic reactivity [22]. In humans, soy dietary foods can potentially reduce ischemic heart disease through multiple mechanisms, including improvement of endothelial function [23].

Exercise and physical activity is well accepted as a preventative method for many non-communicable diseases such as type 2 diabetes and CVD. The role of physical activity becomes more important through menopause because exercise can counter the associated increased risk for CVD, osteoporosis, musculoskeletal symptoms, depression, and obesity [24, 25].

Recent research indicates that a combinatory regimen of isoflavone supplementation and exercise may be beneficial to menopausal women through modulating the hepatic protein expression profile [26]. This study also indicated that the combination of an isoflavone diet and exercise was more effective in reversing changes in ovariectomy-induced hepatic protein expression than either intervention alone. Furthermore, the combination of exercise and a soy isoflavone diet was shown to have beneficial effects in terms of protecting against cardiovascular risk factors by controlling lipid profiles and the related enzyme, paraoxonase PON, as well as NO activity and apoptosis of the aorta in OVX rats [27]. In contrast, an animal study revealed that administration of genistein, treadmill running, or the combination of both, was not associated with any improvement in cardiovascular function and structure or risk factors in an ovariectomy model of postmeno- pause [28]. However, these studies do not focus on vascular endothelial apoptosis and its related factors induced by ovariectomy. Thus, we undertook to investigate the effects of combined resistance/endurance exercise (RE) and/or soybean supplementation in a rat model of menopause (ovariectomy). We hypothesized that soy isoflavone supplementation in combination with $\mathrm{RE}$ training could modulate the menopause-induced changes in eNOS expression in the heart of ovariectomized rats to a greater extent than either soy extract supplementation or RE alone.

\section{Material and methods}

\section{Animals}

All procedures were approved by the Committee on Animal Experimentation and Ethics of the University of Tehran (Process Number: 2017/ 5200690, Date of Approval: February 1, 2017). Fifty healthy female Wistar rats (body weight, $200 \pm 25 \mathrm{~g}$ ) were provided from the Experimental Animal Research Center, Faculty of Veterinary, Islamic Azad University, Tabriz, Iran. The experimental animals received humane care. The animals were housed in an air-conditioned environment $\left(22 \pm 2^{\circ} \mathrm{C}\right)$, with a 12 -h light/dark cycle (7:0019:00) and were allowed free access to standard (basal) diet and water throughout the experiment. After a 1-week acclimation period, the rats were surgically ovariectomized (OVX) and continued to receive a basal diet during the week following surgery and were randomly divided into five groups ( $n=10)$ as follows: 1) SHAM (rats were incised and sutured without removal of the ovaries); 2) OVX (rats underwent removal of the bilateral ovaries); 3) OVX + SOY (rats underwent OVX + soy isoflavone supplementation); 4) OVX + RE (rats underwent OVX + combined resistance/endurance training); 5) OVX + SOY + RE (rats underwent OVX + soy isoflavone supplementation + combined resistance/endurance training).

After 2 weeks of recovery the animals in OVX + SOY and OVX + SOY + RE groups received $60 \mathrm{mg} /$ $\mathrm{kg}$ of soy extract oral gavage daily for 6 weeks superimposed on their basal diet. The animal diets were prepared using modified American Institute of Nutrition (AIN)-76.

\section{Exercise training protocol}

The exercise training combined the two endurance and resistance models described below. The exercise sessions were performed in a continuous sequence (resistance and endurance training) for 6 weeks (five times per week).

During the first week, the animals in the OVX + $\mathrm{SOY}+\mathrm{RE}$ and $\mathrm{OVX}+\mathrm{RE}$ groups were accustomed to running on a motor-driven treadmill at $10 \mathrm{~m} / \mathrm{min}$, 
without an incline for $11 \mathrm{~min} /$ day for 5 days, as described previously [29]. After this period, all rats ran 5 days/week for 6 weeks. The running protocol was as follows: week $1=15 \mathrm{~m} / \mathrm{min}$ for $25 \mathrm{~min}$; week $2=16 \mathrm{~m} / \mathrm{min}$ for $32 \mathrm{~min}$; week $3=17 \mathrm{~m} /$ $\min$ for $39 \mathrm{~min}$; week $4=18 \mathrm{~m} / \mathrm{min}$ for $46 \mathrm{~min}$; week $5=53 \mathrm{~m} / \mathrm{min}$; and week $6=20 \mathrm{~m} / \mathrm{min}$ for 60 min.

Resistance training was performed for 6 weeks as previously described [30]. A ladder $(110 \mathrm{~cm}$ high, $18 \mathrm{~cm}$ wide, $2 \mathrm{~cm}$ space between steps) was placed at an $80^{\circ}$ inclination, and a resting chamber $(20 \times 20 \times 20 \mathrm{~cm})$ was placed on the top of the ladder. The animals climbed the ladder carrying weights attached to the base of the tail, and the weights were gradually increased with exercise progression. Familiarization of the animals with the exercise apparatus was performed during 3 consecutive days with nine repetitions per day. Training sessions consisted of one set of climbing, using progressive loads, interspaced with 90-second intervals. The animals had to perform 8-10 repetitions to go from the base to the top of the ladder. The load was progressively increased from $50 \%$ of the maximum load in the first series, through $75 \%, 90 \%$ until $100 \%$ of the maximum load in the fourth series. After that, resistance was increased in increments of $30 \mathrm{~g}$ per attempt until failure. Training was performed in the afternoon, for five times a week, from Monday to Friday.

\section{Ovariectomy surgery}

For the ovariectomy, the animals were anesthetized with a solution of ketamine $(100 \mathrm{mg} / \mathrm{kg}$, ip) and xylazine $(10 \mathrm{mg} / \mathrm{kg}$, ip). The ovariectomy was preceded by a mid-line dorsal skin incision, and each ovary and a part of the oviduct was removed. After surgery, the muscles and the skin were sutured and the animals were kept warm. In the SHAM group, the same surgical procedures were performed as those for the OVX groups, but the ovaries were not removed.

\section{Soy extracts}

Soy was procured from Tabriz City, Iran, and was scientifically identified by the Department of Botany of University of Tabriz, Iran, and the voucher specimen of the soybean was deposited. To prepare the hydroalcoholic extract, $50 \mathrm{~g}$ of the crumbled, dried plant was extracted with $300 \mathrm{ml}$ of ethanol-water $(70 / 30, v / v)$, using a rotary vacuum evaporator in order to reduce the dryness of the extracts [31].

\section{RNA isolation and cDNA synthesis}

Rats were sacrificed and hearts excised at the end of the six-week training period. As previously described [32], total RNA was extracted from the left ventricle of the heart using the RNX-Plus solution kit (Geneall, Cinagen, RiboEX LS, Republic of Korea, cat $\neq 305$-101) according to the manufacturer's instructions. RNA quantity and A260/280 ratio were measured using NanoDrop 1000 (Thermo Scientific, Waltham, and Mass), and gel electrophoresis with GelRed (Biotium, Hayward, California) was used to evaluate the integrity of each RNA sample. eNOS gene expression was quantitatively assessed by real-time polymerase chain reaction. The following primer pairs were used: 5'-AAACTCTGCAGCCAGCTCTTG-3' (sense) and 5'-GACCTCCCACTTTGCGTTTGA-3' (antisense) for rat eNOS. PCR was performed with $18 \mathrm{~S}$ rRNA as an internal standard [33]. The eNOS expression was normalized to Gaph expression using the following primer pairs: 5'-CAAGATCATCAGCAATGCCTCC-3' (Sense) and 5'-GCCATCACGCCAGTTTCC-3'. For synthesis of cDNA, $1 \mu$ lof total RNA $(200 \mathrm{ng} / \mu \mathrm{l})$ was reverse transcribed by Revert Aid MMuLV reverse transcriptase $(1 \mu \mathrm{l})$, DNase I $\left(\begin{array}{ll}1 & \mu l\end{array}\right)$ and random hexamer primers $(1 \mu \mathrm{l})$, dNTPS $(2 \mu \mathrm{l})$, and RiboLock RNase-inhibitor $(0.25 \mu \mathrm{l})$, for $10 \mathrm{~min}$ at $25^{\circ} \mathrm{C}$, followed by $60 \mathrm{~min}$ at $42^{\circ} \mathrm{C}$ in a final volume of $20 \mu \mathrm{l}$. The reaction was terminated by heating at $70^{\circ} \mathrm{C}$ for $5 \mathrm{~min}$.

\section{Statistical analysis}

Data were analyzed using the Shapiro-Wilk test, which confirmed the Gaussian distribution of the data, after which one-way ANOVA followed by Tukey's post-test was used to analyze the differences between the groups. The tests were performed using SPSS 17 software with an adopted significance level of $p<0.05$.

\section{Results}

\section{Body weight and food intake}

As shown in Table I, the OVX group had a significantly higher body weight after 6 weeks compared to the SHAM group ( $p \leq 0.05)$. SOY and/or exercise $(\mathrm{RE}+\mathrm{EN})$ treatment returned body weight to baseline as there was no difference between these groups and SHAM. There was a difference in body weight between the OVX group and OVX $+R E+E, O V X+S O Y$ and OVX + RE + EN + SOY groups. There were no significant differences in daily food intake between the groups.

\section{Effects of various treatments on eNOS gene expression in the heart}

As shown in Figure 1 the expression of eNOS in the ventricle was significantly lower in OVX compared to SHAM $(p \leq 0.05)$. Treatment with either SOY or RE (OVX + RE and OVX + SOY) resulted in 
Table I. Food intake and body weights of the rats during the study period

\begin{tabular}{|lccccc|}
\hline Groups & SHAM & OVX & OVX + SOY & OVX + RE + EN & OVX + SOY + RE + EN \\
\hline $\boldsymbol{N}$ & 10 & 10 & 10 & 10 & 10 \\
\hline BW final [g] & $264.1 \pm 2.6$ & $283.2 \pm 4.2^{*}$ & $265.4 \pm 5.3$ & $263.7 \pm 3.5$ & $264.2 \pm 4.2$ \\
\hline $\begin{array}{l}\text { Food intake } \\
\text { [g/day] }\end{array}$ & $16.43 \pm 2.16$ & $16.13 \pm 3.62$ & $15.93 \pm 3.62$ & $15.84 \pm 2.12$ & $16.03 \pm 3.21$ \\
\hline
\end{tabular}

$B W$ - body weight, OVX - ovariectomized group, OVX + RE - ovariectomized with 6-week RE training group, OVX + SOY - ovariectomized with 6-week soy extract supplementation group, $O V X+R E+S O Y$ - ovariectomized with 6-week soy extract supplementation and RE training group. Values are means \pm SEM $(n=10)$. ${ }^{*} P<0.05$ SHAM vs. other groups.

significantly higher eNOS expression of the ventricle compared to the OVX group ( $p \leq 0.05)$. Combined treatment (OVX + SOY + RE) further increased eNOS expression compared to individual treatments $(p<0.05)$.

\section{Discussion}

Postmenopausal women frequently experience body weight gain and become more vulnerable to developing obesity-related diseases such as CVD, hypertension and type 2 diabetes mellitus [6, 34]. However, other distinct effects of the low estrogen state associated with menopause may also increase the risk of developing CVD and diabetes.

This study was designed to investigate the effects of exercise and soybean supplementation on the menopause-induced reduction of a marker of vascular function. We hypothesized that soy, in combination with RE training, could reverse the menopause-induced changes in eNOS expression in the heart of ovariectomized rats to a greater extent than either soy or RE alone.

Firstly, the ovariectomy model proved successful in reducing eNOS expression, confirming a relationship between this menopause model and vascular function, in rodents at least. Our data confirm our hypothesis that soy isoflavone supplementation in combination with resistance-endurance training can reverse the ovariectomy-induced changes in eNOS expression in the heart of ovariectomized rats. Greater eNOS activity in cardiac muscle of rodents suggests improved vascular tone and has atheroprotective effects. Certainly, interventions associated with reduced risk factors also show increased eNOS activity [35].

Our results thus further indicate that the combination of soy extract supplementation and regular resistance-endurance (RE) training has a greater cardiac protective effect than employing either strategy alone.

In regard to human-based research, there is evidence that soybean-based polyphenol supplementation can benefit lipid oxidation and subsequently endurance exercise performance $[36,37]$ and, further, benefit CVD risk factors in menopausal women through modulating hepatic pro-

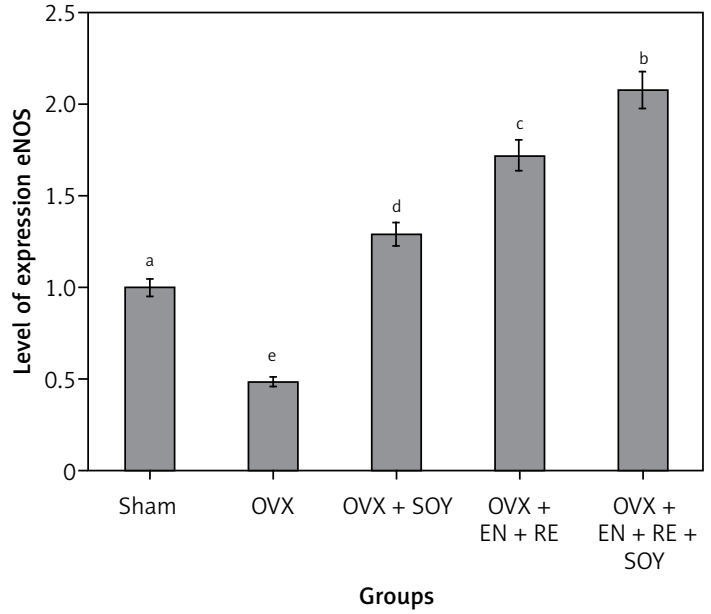

Figure 1. Level of eNOS expression in heart of OVX rats in different treatment groups

OVX - ovariectomized group, $O V X+R E$ - ovariectomized with 6-week RE training group, OVX + SOY-ovariectomized with 6-week soy extract supplementation group, $O V X+R E+S O Y-$ ovariectomized with 6-week soy extract supplementation and RE training group. eSignificant difference compared with SHAM group ( $p \leq 0.05$ ), ${ }^{d}$ significant difference compared with other groups $(p<0.05)$, ssignificant difference compared with other groups ( $p \leq 0.05)$, ${ }^{b}$ significant difference compared with other groups $(p \leq 0.05)$. Data are expressed as mean \pm SEM.

tein expression profiles [26]. Exercise, of course, is well known to improve cardiovascular disease risk in humans and rodent models. Whilst this study was performed in rodents only, and measured only a marker of vascular function (eNOS expression), the data warrant the investigation of soy and exercise prescription together as a prevention strategy for the increased risk of CVD associated with menopause in humans. That the effects of RE training and soy were additive suggests, however, that (soy) isoflavone and exercise interventions together may be a more powerful strategy for reducing post-menopausal CVD risk than either exercise or diet alone. That exercise has considerable benefits for CVD prevention makes such an intervention cost effective, and that soy is a simple and cheap food provides additional incentive for this primary preventative combination. Future research should attempt to identify whether our observations on eNOS expression translate into 
measureable physiological effects on vascular function, particularly in skeletal muscle.

In conclusion, the current study shows that a combination of soy extract supplementation and regular RE training has a greater beneficial effect on cardiac eNOS activity than either exercise or soy alone. From this we can speculate that this combined approach could produce a better cardiac protective effect in a menopause rat model than either exercise or soy supplementation alone.

\section{Conflict of interest}

The authors declare no conflict of interest.

\section{References}

1. Hongwei S, Jie Y, Hongling J, Hazel L, Dongmin L. Phytoestrogen genistein up-regulates endothelial nitric oxide synthase expression via activation of CAMP response element-binding protein in human aortic endothelial cells. Endocrinology 2012; 153: 3190-8.

2. Huang PL, Huang Z, Mashimo $\mathrm{H}$, et al. Hypertension in mice lacking the gene for endothelial nitric oxide synthase. Nature 1995; 377: 239-42.

3. Lake-Bruse KD, Faraci FM, Shesely EG, Maeda N, Sigmund CD, Heistad DD. Gene transfer of endothelial nitric oxide synthase (eNOS) in eNOS-deficient mice. Am J Physiol 1999; 277: H770-6.

4. Hayashi T, Sumi D, Juliet PA, et al. Gene transfer of endothelial NO synthase, but not eNOS plus inducible NOS, regressed atherosclerosis in rabbits. Cardiovasc Res 2004; 61: 339-51.

5. Nasreen S, Nabika T, Shibata H, et al. T-786C polymorphism in endothelial NO synthase gene affects cerebral circulation in smokers: possible gene-environmental interaction. Arterioscler Thromb Vasc Biol 2002; 22: 605-10.

6. Jin L, Hyung SC, Dae YK, et al. Combined effects of exercise and soy isoflavone diet on paraoxonase, nitric oxide and aortic apoptosis in ovariectomized rats. Appetite 2012; 58: 462-9.

7. Kudou S, Fluery Y, Welti D, et al. Malonyl isoflavone glycosides in soybean seeds (Glycine max Merrill). Agric Biol Chem 1991; 55: 2227-33.

8. Scuteri A, Ferrucci L. Blood pressure, arterial function, structure, and aging: the role of hormonal replacement therapy in postmenopausal women. J Clin Hypertens (Greenwich) 2003; 5: 219-25.

9. Stampfer MJ, Colditz GA. Estrogen replacement therapy and coronary heart disease: a quantitative assessment of the epidemiologic evidence. Prev Med 1991; 20: 47-63.

10. Rossouw JE, Anderson GL, Prentice RL, et al.; Writing Group for the Women's Health Initiative Investigators. Risks and benefits of estrogen plus progestin in healthy postmenopausal women: principal results from the Women's Health Initiative randomized controlled trial. JAMA 2002; 288: 321-33.

11. Akiyama T, Ishida J, Nakagawa S, et al. Genistein, a specific inhibitor of tyrosine-specific protein kinases. J Bio Chem 1987; 262: 5592-5.

12. Antioxidative activity of fermented soybean products. Esaki H, Onozaki H, Osawa T (eds). Vol. I. Fruits and vegetables. American Chemical Society, Washington, D.C. 1994; 1-546.
13. Hamlin GP, Soares MJ. Regulation of deoxyribonucleic acid synthesis in proliferating and differentiating trophoblast cells: involvement of transferrin, transforming growth factor-beta, and tyrosine kinases. Endocrinology 1995; 136: 322-1.

14. Hashimoto N, Ogashiwa M, Iwashita S. Role of tyrosine kinase in the regulation of myogenin expression. Eur J Biochem 1995; 227: 379-87.

15. Ji S, Mills GM, Frank GR, Cornelius SG, Spurlock ME. Soybean genistein inhibits myoblast proliferation, differentiation, and myotube protein synthesis. J Anim Sci 1997; 75 (Suppl. 1): 56.

16. Joannou GE, Kelly GE, Reeder AY, Waring M, Nelson C. A urinary profile study of dietary phytoestrogens. The identification and mode of metabolism of new isoflavonoids. J Steroid Biochem Mol Biol 1995; 54: 167-84.

17. Wei H, Wei L, Frenkel K, Bowen R, Barnes S. Inhibition of tumor promoter-induced hydrogen peroxide formation in vitro and in vivo by genistein. Nutr Cancer 1993; 20: 1-12.

18. Magee PJ, Rowland IR. Phyto-oestrogens, their mechanism of action: current evidence for a role in breast and prostate cancer. Br J Nutr 2004; 91: 513-31.

19. Park OJ, Surh YJ. Chemopreventive potential of epigallocatechin gallate and genistein: evidence from epidemiological and laboratory studies. Toxicol Lett 2004; 150: 43-56.

20. Sarkar FH, Li Y. Soy isoflavones and cancer prevention. Cancer Invest 2003; 21: 744-57.

21. Wang W, Higuchi CM, Zhang R. Individual and combinatory effects of soy soflavones on the in vitro potentiation of lymphocyte activation. Nutr Cancer 1997; 29: 29-34.

22. Duncan AM, Underhill KE, Xu X, Lavalleur J, Phipps WR, Kurzer MS. Modest hormonal effects of soy isoflavones in postmenopausal women. J Clin Endocrinol Metab 1999; 84: 3479-84.

23. Kannel WB, Wilson PW. Risk factors that attenuate the female coronary disease advantage. Arch Intern Med 1995; 155: 57-61.

24. Messina M. Soy foods, isoflavones, and the health of postmenopausal women. Am J Clin Nutr 2014; 100 (suppl): 423S-30S.

25. Al-Nakkash L, Martin JB, Petty D, et al. Dietary genistein induces sex-dependent effects on murine body weight, serum profiles, and vascular function of thoracic aortae. Gender Med 2012; 9: 295-308.

26. Sun Y, Joomin L, Seung ML. Combined treatment of isoflavone supplementation and exercise restores the changes in hepatic protein expression in ovariectomized rats - a proteomics approach. J Int Soc Sports Nutr 2014; 11: 29.

27. Ou PF, Zhang L. Stimulatory effects of soybean isoflavones on exercise performance. Int J Phys Sci 2010; 5: 2272-7.

28. Jin L, Hyung SC, Dae YK, et al. Combined effects of exercise and soy isoflavone diet on paraoxonase, nitric oxide and aortic apoptosis in ovariectomized rats. Appetite 2012; 58: 462-9.

29. Al-Nakkash L, Janjulia T, Peterson K, et al. Genistein and exercise do not improve cardiovascular risk factors in the ovariectomized rat. Climacteric 2014; 17: 136-47.

30. Oh HY, Lim S, Lee JM, Kim DY, Ann ES, Yoon S. A combination of soy isoflavone supplementation and exercise improves lipid profiles and protects antioxidant defense-systems against exercise-induced oxidative stress in ovariectomized rats. Biofactors 2007; 29: 175-85. 
31. De Cássia Cypriano Ervati Pinter R, Padilha AS, de Oliveira EM, Vassallo DV, de Fúcio Lizardo JH. Cardiovascular adaptive responses in rats submitted to moderate resistance training. Eur J Appl Physiol 2008; 103: 605-13.

32. Mohammadpour T, Hosseini $M$, Karami $R$, Sadeghnia HR, Ebrahimzadeh Bideskan AR, Enayatfard L. Estrogen-dependent effect of soy extract on pentylenetetrazole-induced seizures in rats. Zhong $\mathrm{Xi}$ Yi Jie He Xue Bao 2012; 10: 1470-6.

33. Felaco M, Grilli A, Gorbunov N, et al. Endothelial NOS expression and ischemia-reperfusion in isolated working rat heart from hypoxic and hyperoxic conditions. Biochim Biophys Acta 2000; 1524: 203-11.

34. Innis M, Gelfand D, Sninsky J, White T. PCR Protocols, a Guide to Methods and Applications. Academic Press, San Diego 1990; 1-482.

35. Melton S, Hegsted M, Keenan MJ, et al. Swimming eliminates the weight gain and abdominal fat associated with ovariectomy in the retired breeder rat despite high-fat diet selection. Appetite 2000; 35: 1-7.

36. Meng C, Liu J, Du AL. Cardio-protective effect of resveratrol on atherogenic diet-fed rats. Int J Clin Exp Pathol 2014; 7: 7899-906.

37. Yeh TS, Chan KH, Hsu MC, Liu JF. Supplementation with soybean peptides, taurine, Pueraria isoflavone, and ginseng saponin complex improves endurance exercise capacity in humans. J Med Food 2011; 14: 219-25. 